\title{
Modelización de la demanda de energía eléctrica: más allá de la normalidad
}

\author{
RENDÓN, JUAN F. \\ Institución Universitaria ITM (Colombia) \\ Correo electrónico: juanrendon@itm.edu.co \\ TREspalacios, AlfREdo \\ Institución Universitaria ITM (Colombia) \\ Correo electrónico: alfredotrespalacios@itm.edu.co \\ CORTÉS, LINA M. \\ Universidad EAFIT (Colombia) \\ Correo electrónico: 1cortesd@eafit.edu.co \\ VILLADA-MEDINA, HERNÁN D. \\ Institución Universitaria ITM (Colombia) \\ Correo electrónico: hernanvillada@itm.edu.co
}

\begin{abstract}
RESUMEN
Este trabajo propone un modelo de demanda de energía eléctrica basado en métodos de series de tiempo y estadística semi-noparamétrica (SNP). Esto permite conocer no solo el valor esperado de la demanda sino también su distribución de probabilidad de manera que, mediante el cálculo de métricas como la Medida de Riesgo de Cuantil (Quantile Risk Metrics), se puedan tomar decisiones basadas en valores extremos menos o más favorables que el valor esperado. Los resultados muestran que para el caso de la demanda de energía eléctrica del mercado colombiano entre los años 2000 y 2018 la distribución de probabilidad de la demanda diaria promedio es leptocúrtica. Es decir, los eventos extremos ocurren con mayor frecuencia que aquellos que suponen una distribución normal. De modo que, el supuesto de distribución gaussiana conlleva a la subvaloración del riesgo en términos de la subvaloración de la frecuencia de valores extremos.
\end{abstract}

Palabras clave: demanda de energía, modelización semi-noparamétrica, mercado de energía, medida de riesgo de cuantil.

Clasificación JEL: C58; Q42.

MSC2010: 41A58; 60E05; 60G70. 


\title{
Electrical energy demand modeling: beyond normality
}

\begin{abstract}
This work proposes a model of electrical energy demand based on time series methods and semi-nonparametric statistics (SNP). This allows knowing not only the expected value of the demand but also its probability distribution so that, by calculating metrics such as the Quantile Risk Metrics, decisions can be made based on less or more extreme values favorable than the expected value. The results show that in the case of electricity demand in the Colombian market between 2000 and 2018, the probability distribution of the average daily demand is leptokurtic. That is, extreme events occur more frequently than those assumed by a normal distribution. Thus, the Gaussian distribution assumption leads to undervaluation of risk in terms of undervaluation of the frequency of extreme values.
\end{abstract}

Keywords: energy demand, semi-nonparametric modelling, energy market, quantile risk metrics.

JEL classification: C58; Q42.

MSC2010: 41A58; 60E05; 60G70.

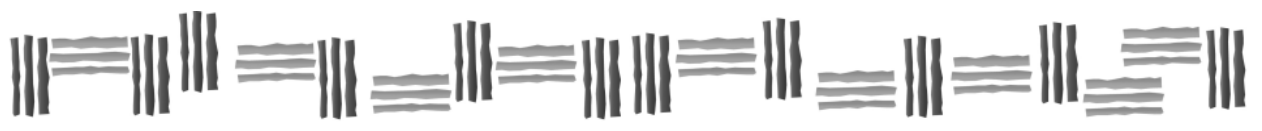




\section{Introducción.}

La principal característica que diferencia los mercados de electricidad de otros mercados corresponde con la necesidad de producir la energía en el mismo instante que ésta es consumida, a tal punto que en tiempo real los sistemas deben mantener un perfecto balance: en cada momento la demanda de energía eléctrica es igual a la generación. Esta particularidad impide, por ejemplo, el arbitraje intertemporal por parte de quienes realizan transacciones en este mercado. El análisis de la demanda de energía eléctrica es importante para comprender no solo los patrones de consumo de los usuarios y su relación con los ciclos económico y climático sino también para identificar uno de los elementos que afecta la formación de precio, tanto spot (al contado o bolsa de energía) como en contratos de venta a futuro (contratos de largo plazo o contratos forward) (Lucia \& Schwartz, 2002).

De acuerdo con Domeett (2015), la identificación de la dinámica de la demanda de energía eléctrica permite a los agentes privados y estatales la planificación tanto de la producción del país como de las políticas públicas. El objetivo de dichas políticas es lograr la sostenibilidad de las empresas y la satisfacción de las necesidades de la población en el corto, medio y largo plazo como cuestión de seguridad energética. Tal es su relevancia, que el estudio de la seguridad energética se aborda desde la economía, la estadística, la política, la ingeniería, el medio ambiente y la ciberseguridad. Tópicos sobre los que recaen diferentes tipos de metodologías y herramientas tanto de corte cuantitativo como cualitativo (UPME, 2016).

Desde la economía y la estadística, es común encontrar modelización de tipo econométrico para abordar el tratamiento de las series de la demanda de energía eléctrica. Dicha modelización, usualmente, es realizada considerando que la incertidumbre de las variables está explicada por una distribución normal (definida por un parámetro de ubicación y otro de dispersión), que carece de sesgo y cuya curtosis se mantiene siempre constante. Sin embargo, considerar que la distribución normal logra describir la incertidumbre de la demanda de energía puede limitar los hallazgos de los investigadores o llegar a conclusiones que no obedecen a la realidad de los datos.

El objetivo de este estudio es proponer un modelo de demanda de energía eléctrica basado en métodos de series de tiempo y estadística semi-noparamétrica (SNP), que permita conocer no solo el valor esperado de la demanda sino también su distribución de probabilidad de manera que mediante el cálculo de medidas la Medida de Riesgo de Cuantil (Quantile Risk Metrics) se puedan tomar decisiones basadas en valores extremos menos o más favorables que el valor esperado. Como caso de estudio se considera la demanda de energía eléctrica en el mercado colombiano entre los años 2000 y 2018.

Este trabajo se desarrolla de la siguiente manera. En la segunda sección se presentan los antecedentes del estudio de la demanda de energía eléctrica. La tercera sección describe la metodología empleada para modelizar la demanda de energía eléctrica. En la cuarta sección se presentan los resultados obtenidos y, finalmente, en la última sección recopilamos las conclusiones del trabajo.

\section{Antedecedentes.}

La modelización de la demanda de energía eléctrica es un tema de interés para los investigadores sobre la gestión del riesgo de mercado a lo largo de la planificación, construcción y operación de sistemas energéticos. Ioannou, Angus y Brennan (2017) presentan una revisión de 161 artículos sobre los métodos cuantitativos y semicuantitativos que se han utilizado para modelizar riesgos e incertidumbres en la planificación de sistemas de energía sostenible y los estudios de viabilidad, incluida la construcción de portafolios óptimos de tecnología energética. El estudio muestra que la demanda de energía es modelizada generalmente a través de métodos probabilísticos, asumiendo que sigue una función de distribución normal de probabilidad. 
Dentro de los métodos de análisis estadístico para la proyección de la demanda se encuentran los trabajos que emplean modelos de regresión lineal como Melikoglu (2013), Callaway (2010), Bianco, Manca y Nardini (2009), Andalib y Atry (2009), González-Romera et al. (2006) y Bentzen y Engsted (1993). Los métodos de regresión utilizan datos de series temporales para estimar la demanda de energía, pero no analizan los datos para establecer sus propiedades. Esto lleva a suponer, implícitamente, que los datos son estacionarios, lo que significa que sus medias y variaciones no varían sistemáticamente con el tiempo. El uso del análisis de cointegración permite el uso de datos sobre variables no estacionarias para estimar coeficientes siempre que las variables estén cointegradas, es decir, que tienen una relación de equilibrio a largo plazo. Dentro de los trabajos que emplean cointegración en el análisis de la demanda de energía se encuentran Engle et al. (1989), Bentzen y Engsted (1993), Bentzen y Engsted (2001), Fouquet et al. (1993), Hunt y Witt (1995), Beenstock, Goldin y Nabot (1999), Nasr, Badr y Dibeh (2000).

Los modelos univariados de series de tiempo aparecen como una alternativa de la predicción de la demanda que no necesita de variables explicativas y del pronóstico de éstas, lo que hace que la incertidumbre del pronóstico sea mayor. El modelo autorregresivo integrado de promedio móvil (ARIMA, por sus siglas en inglés) es una de las técnicas más trabajadas por los investigadores en el pronóstico de la demanda de energía (Debnath \& Mourshed, 2018). El-Desouky y El-Kateb (2000) usaron modelos híbridos de redes neuronales artificiales (ANN, por sus siglas en inglés) y ARIMA para pronosticar la demanda de electricidad para la ciudad de Jeddah en Arabia Saudita encontrando un mejor ajuste que el método oficial de series de tiempo. Erdougdu (2007) utilizó un modelo ARIMA para analizar la demanda de electricidad para Turquía. Los resultados mostraron un buen ajuste a la muestra y sugieren que el modelo oficial está sobrestimando la demanda. Un estudio similar para Turquía realizado por Ediger y Akar (2007) empleó modelos ARIMA para pronosticar la demanda de energía primaria de 2005 a 2020. Yuan et al. (2016) realizaron un pronóstico del consumo de energía primaria en China de 2014 a 2020 mediante el uso de modelos ARIMA y modelos grises (GM, por sus siglas en inglés). Los resultados mostraron que si bien el crecimiento de la demanda esperada es alto sería menor que en la primera década del siglo XXI. Hussain et al. (2016) utilizaron un modelo ARIMA para pronosticar la demanda del consumo total y por sectores de electricidad en Pakistán hasta el 2020. Se encontró que el crecimiento esperado en el sector residencial era particularmente alto y que de cumplirse el pronóstico la demanda superaría a la oferta.

Pese a que los modelos de demanda anteriormente mencionados han sido ampliamente utilizados, en general las conclusiones y las comparaciones que se hacen con otros modelos están basados generalmente en el valor esperado del respectivo modelo. Pero es sabido que desde un punto de vista de la gestión de riesgo es necesario conocer, no solo el valor esperado de la demanda, sino también su distribución de probabilidad de manera que mediante el cálculo de medidas como la Medida de Riesgo de Cuantil (Quantile Risk Metrics), se puedan tomar decisiones basadas en valores menos o más favorables que el valor esperado (Ioannou et al., 2017).

Los modelos de regresión lineal y los modelos ARIMA proporcionan una distribución normal de probabilidad que permiten calcular intervalos de confianza y percentiles, pero como se presenta más adelante en este estudio, los datos empíricos de la demanda de electricidad en el caso colombiano presentan asimetría o la recurrencia de eventos extremos que resulta en distribuciones de cola pesada. Como resultado de la interacción de las variables involucradas, la demanda de la energía eléctrica exhibe características de estacionalidad, reversión a la media y colas pesadas. Tales características hacen pensar que la suposición de normalidad puede ser insuficiente para representar la incertidumbre de la demanda de electricidad.

En los últimos años, varios estudios han intentado superar las limitaciones del supuesto común de normalidad sobre el rendimiento del precio de las acciones, commodities y variables del mercado de electricidad (León et al., 2007; Trespalacios et al., 2020), que a menudo se rechaza en la literatura financiera empírica, incluso después de controlar los efectos de agrupamiento (clusters) de volatilidad. 
Aunque esta suposición aún puede ser razonable si el interés se centra en los dos primeros momentos condicionales (Bollerslev \& Wooldridge, 1992), en muchas aplicaciones financieras las características en estudio involucran momentos de orden superior, como la asimetría y la curtosis. Desafortunadamente, la normalidad es demasiado restrictiva para aproximar la forma compleja de la distribución de la demanda de electricidad y se requiere de distribuciones más flexibles para explicar su comportamiento y tomar decisiones más acertadas.

Recientemente, la aplicación de modelos de regresión SNP en el pronóstico de la demanda, ha recibido cada vez más atención y numerosas publicaciones ilustran su desempeño superior. Por ello, en la presente investigación se aborda la modelización de la demanda de energía eléctrica desde la estadística SNP, como una opción que permite capturar la incertidumbre de variables aleatorias sin limitarlas solo a dos primeros momentos de la distribución. Algunos estudios que usan modelos de tipo SNP para el tratamiento de series con colas pesadas y sesgo son presentados por Brunner (1992), Mauleon y Perote (2000), Ñíguez y Perote (2011), Cortés, Mora-Valencia y Perote (2018), Jondeau y Rockinger (2001).

\section{Metodología y datos.}

\subsection{Modelo de demanda diaria promedio.}

Se propone un modelo de demanda que tiene el objetivo de capturar patrones en el tiempo, como son la tendencia, efectos estacionales mensuales y efectos AR y MA. En este modelo la única variable exógena es el tiempo. En este modelo la demanda $D_{t}$ tiene dos componentes: una función determinística $\mathrm{f}(\mathrm{t})$ que depende del tiempo y un error aleatorio $e_{t}$. La función determinística está compuesta por un conjunto de variables dummy que explica efectos mensuales y un conjunto de parámetros AR y MA que explican otros efectos estacionales.

$$
\begin{gathered}
D_{t}=f(t)+e_{t} \\
\mathrm{f}(\mathrm{t})=\beta_{0}+\sum_{m=1}^{12} \beta_{m} \cdot M_{m}+\beta_{\text {tend }} \cdot t+\sum_{i=1}^{p} \psi_{i} \cdot D_{t-i}-\sum_{j=1}^{q} \theta_{j} \cdot e_{t-j}, \\
\frac{e_{t}}{\sigma} \sim g(0,1, S, K) .
\end{gathered}
$$

$\beta_{\text {tend }}$ es el parámetro que mide la tendencia, $M_{m}$. Son variables dummies que explican efectos mensuales y $\beta_{m}$. El conjunto de parámetros que miden estos efectos. $\psi_{i}$ es un conjunto de parámetros AR y $D_{t}$ el conjunto de variables rezagadas. $\theta_{j}$ es un conjunto de parámetros MA y $e_{t}$ el conjunto de errores rezagados.

$g(*)$ es una función de densidad de probabilidad Gram-Charlier tipo A con media cero, varianza 1 y un conjunto de parámetros de $S$ y $K$ que parametrizan el sesgo y la curtosis respectivamente.

\section{i. Modelo de densidad de probabilidad Gram - Charlier}

Una función de densidad de probabilidad $g$ de tipo SNP truncada en $n$ términos que caracteriza una variable aleatoria estandarizada $x$, se describe en la ecuación (1). Donde $\mathbf{d}$ corresponde con un vector de parámetros $d_{i}, \phi(x)$ es la función de densidad de probabilidad (pdf) de la distribución normal estándar de la ecuación (2) y $H_{i}(x)$ que se muestra en (3), es el polinomio de Hermite de orden $i$.

$$
g(x ; \boldsymbol{d})=\left[1+\sum_{s=1}^{n} d_{s} \cdot H_{s}(x)\right] \phi(x),
$$




$$
\begin{aligned}
& \phi(x)=\frac{1}{2 \pi} e^{-\frac{x^{2}}{2}}, \\
& H_{i}(y)=\frac{(-1)^{i}}{\phi(x)} \cdot \frac{d^{i} \phi(x)}{d x^{i}} .
\end{aligned}
$$

Como se muestra en la ecuación (3), cada uno de los polinomios de Hermite requiere la derivación de orden $i$ de la pdf normal estándar. Los primeros cinco polinomios de Hermite se muestran a continuación:

$$
\begin{aligned}
& H_{0}=1, \\
& H_{1}=x, \\
& H_{2}=x^{2}-1, \\
& H_{3}=x^{3}-3 x, \\
& H_{4}=x^{4}-6 x^{2}+3, \\
& H_{5}=x^{5}-10 x^{3}+15 x .
\end{aligned}
$$

A continuación, se muestra cómo se relacionan los momentos de la distribución en función de cada uno de los parámetros $d_{i}$. Se puede identificar como cada momento $i$ de la variable aleatoria es función del parámetro $d i$ y otros parámetros $d_{j}, j<i$. Así, el número de coeficientes requeridos para describir pdf corresponderá con la cantidad de momentos requeridos para describir los datos obtenidos.

$$
\begin{aligned}
& \mu_{1}=d_{1}, \\
& \mu_{2}=2 d_{2}+1, \\
& \mu_{3}=6 d_{3}+3 d_{1}, \\
& \mu_{4}=24 d_{4}+12 d_{2}+3, \\
& \mu_{5}=120 d_{5}+60 d_{3}+15 d_{1} .
\end{aligned}
$$

El ajuste de distribución de los datos se realiza por el método de máxima verosimilitud descrito por Del Brio y Perote (2012), que busca encontrar el conjunto de parámetros $\theta$ que maximiza la función de log-verosimilitud $l(\theta)$ que se muestra en el problema de optimización que se especifica en (15) y es función del valor de la pdf, $f(\cdot)$, aplicado a cada una de las observaciones $x_{i}$. El vector $\hat{\theta}$ se refiere a los valores estimados en el proceso de ajuste.

$$
\begin{aligned}
& \hat{\theta}=\operatorname{argmax}\{l(\theta)\}, \\
& l(\theta)=\log [L(\theta)]=\sum_{i=1}^{N} \log \left[f\left(x_{i} \mid \theta\right)\right] .
\end{aligned}
$$




\subsection{Muestra y variables.}

Para llevar a cabo este estudio se tomaron datos mensuales del Portal BI del Operador y Administrador del Sistema Eléctrico Colombiano (XM) de la demanda de energía eléctrica (en Gigawatt hora - GWh) del mercado colombiano entre los años 2000 y 2018. El Gráfico 1 y la Tabla 1 presentan la serie de tiempo y algunas estadísticas descriptivas respectivamente para todo el período muestral de la demanda diaria promedio, calculada como la demanda mensual dividida por el número de días del mes. En este grafico se observa una tendencia creciente durante todo el periodo de análisis y patrones estacionales. Por su parte, en la Tabla 1, las estadísticas brindan señales de existencia de valores extremos que pueden llevar a rechazar el supuesto de la normalidad.

Gráfico 1. Demanda diaria promedio.

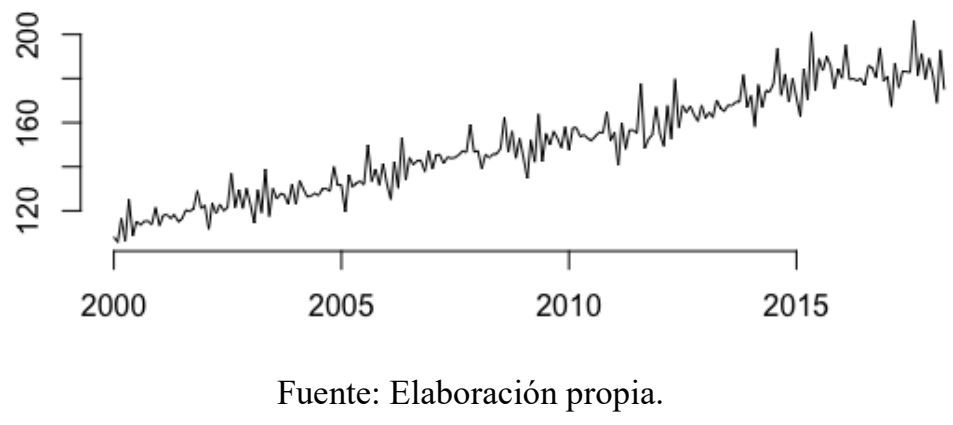

Tabla 1. Estadística descriptiva de la demanda de energía diaria promedio.

\begin{tabular}{lc}
\hline \multicolumn{1}{c}{ Estadístico } & $\begin{array}{c}\text { Demanda de } \\
\text { energía } \\
\text { (GWh) }\end{array}$ \\
\hline \hline Mediana & 148.5 \\
Media & 150.2 \\
Máximo & 206 \\
Mínimo & 105.8 \\
Desv. Estándar & 23.27 \\
Percentil 1 & 108.3 \\
Percentil 5 & 115.3 \\
Percentil 10 & 119.6 \\
Percentil 90 & 181.4 \\
Percentil 95 & 185.9 \\
Percentil 99 & 194.9 \\
\hline
\end{tabular}

Fuente: Elaboración propia con información del Portal BI del Operador y Administrador del Sistema Eléctrico Colombiano, XM.

En el ACF de la Demanda diaria promedio (Gráfico 2a) se puede observar una fuerte autocorrelación. En el ACF de la diferencia (Gráfico 2b) de la Demanda diaria promedio, la correlación es fuerte cuando el retardo es múltiplo de 9 , lo que implica un patrón de comportamiento estacional de la serie Tsay (2005). 
Gráfico 2. Función de autocorrelación de la demanda y su primera diferencia.

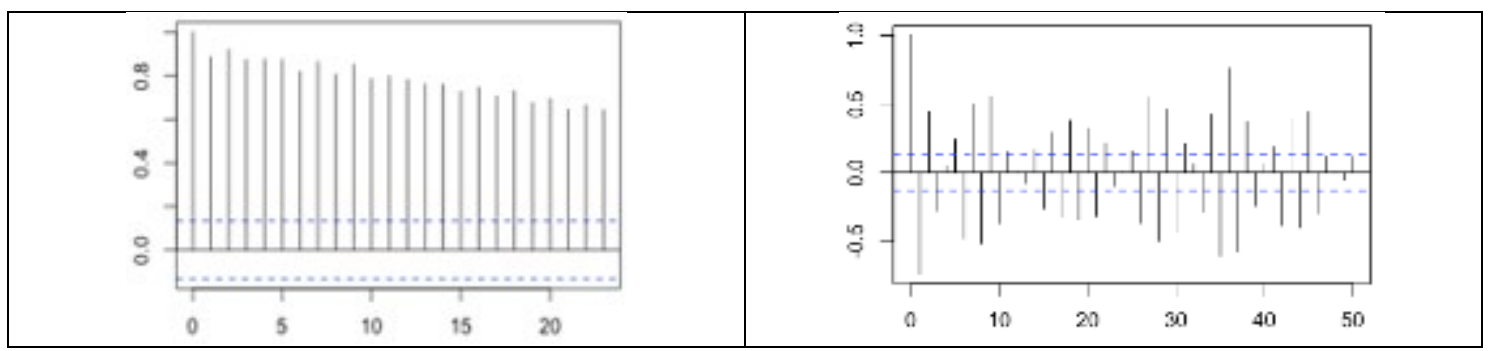

(a)

(b)

Fuente: Elaboración propia.

\section{Resultados.}

\subsection{Resultados modelo demanda de energía.}

En la Tabla 2 se presentan los resultados obtenidos de la estimación de la tendencia y efectos estacionales mensuales. Según los resultados de los valores estimados de los parámetros de las variables categóricas mensuales, no hay una diferencia significativa entre los meses 1, 4 y 6 de la demanda diaria (meses de nivel medio de demanda), el mes 2 tiene un nivel de demanda diaria más bajo que los meses de nivel medio de demanda y el resto de meses son los meses con más altos niveles de demanda diaria (en promedio el mes 8 es el de más alta demanda diaria).

Tabla 2. Estimación de la tendencia y efectos estacionales mensuales.

\begin{tabular}{|c|c|c|c|c|}
\hline & Estimado & Std. Error & $\mathrm{t}$ value & $\operatorname{Pr}(>|t|)$ \\
\hline Intercepto & 89.0289210 & 1.2671287 & 70.260362 & $0.0000000 * * *$ \\
\hline Tendencia & 0.3496544 & 0.0061372 & 56.973318 & $0.0000000 * * *$ \\
\hline Mes 2 & -3.6237371 & 1.5342561 & -2.361886 & $0.0191012 *$ \\
\hline Mes 3 & 6.3592961 & 1.5342758 & 4.144819 & $0.0000494 * * *$ \\
\hline Mes 5 & 8.3674804 & 1.5658406 & 5.343763 & $0.0000002 * * *$ \\
\hline Mes 7 & 4.1688131 & 1.5657590 & 2.662487 & $0.0083601 * *$ \\
\hline Mes 8 & 9.6066036 & 1.5657543 & 6.135448 & $0.0000000 * * *$ \\
\hline Mes 9 & 3.8274248 & 1.5657736 & 2.444431 & $0.0153367^{*}$ \\
\hline Mes 10 & 5.1136866 & 1.5658170 & 3.265826 & $0.0012755^{* *}$ \\
\hline Mes 11 & 4.1424111 & 1.5658844 & 2.645413 & $0.0087792 * *$ \\
\hline Mes 12 & 6.0605822 & 1.5659759 & 3.870163 & $0.0001454 * * *$ \\
\hline
\end{tabular}

Fuente: Elaboración propia.

En el Gráfico 3 se observa la demanda diaria promedio, sin los efectos mensuales y sin tendencia. En este gráfico todavía se observan patrones estacionales, como los que se aprecian en el correlograma presentado en el Gráfico 1. 
Gráfico 3. Demanda diaria promedio desestacionalizada y sin tendencia.

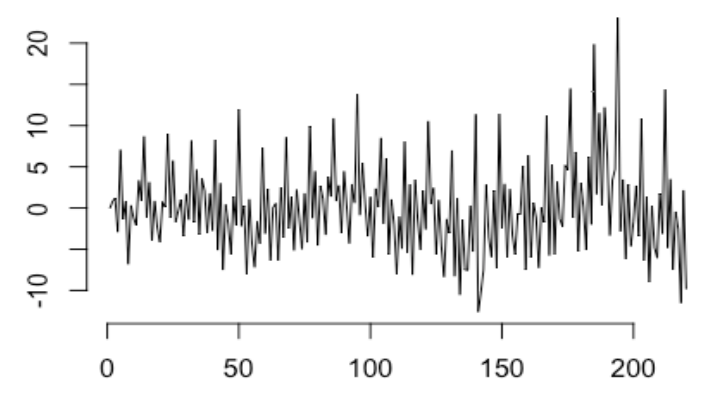

Fuente: Elaboración propia.

En la Tabla 3 se muestra que, en el componente autorregresivo del modelo, resultan significativos los parámetros de los retardos 1, 4 y 9. Para el componente de medias moviles, los parámetros significativos corresponden a los retardos del error 1, 2, 3, 4 y 9.

Tabla 3. Modelo ARMA.

\begin{tabular}{|c|c|c|c|c|}
\hline & Estimado & Std. Error & $\mathrm{t}$ value & $\operatorname{Pr}(>|t|)$ \\
\hline AR1 & -0.049973 & 0.013639 & -3.664 & $0.000248 * * *$ \\
\hline AR4 & -0.022068 & 0.007343 & -3.005 & $0.002653 * *$ \\
\hline AR9 & 0.987256 & 0.012215 & 80.822 & $<2 \mathrm{e}-16 * * *$ \\
\hline MA1 & 0.364965 & 0.054328 & 6.718 & $1.85 \mathrm{e}-11 * * *$ \\
\hline MA2 & 0.313459 & 0.055497 & 5.648 & $1.62 \mathrm{e}-08 * * *$ \\
\hline MA3 & 0.211449 & 0.053000 & 3.990 & $6.62 \mathrm{e}-05 * * *$ \\
\hline MA4 & 0.092886 & 0.037741 & 2.461 & $0.013849 *$ \\
\hline MA9 & -0.722639 & 0.048005 & -15.053 & $<2 \mathrm{e}-16^{* * *}$ \\
\hline AIC & 1088.12 & & & \\
\hline
\end{tabular}

Fuente: Elaboración propia.

La Tabla 4 presenta las estadisticas descriptivas de los residuales del modelo, en donde se estima que la desviación estandar de la demanda diaria promedio es $2.8 \mathrm{GWh}$. La distribución de frecuencias tiene sesgo negativo y es leptocurtica, lo que implica la presencia de colas pesadas. El test de curtosis prueba que el exceso de curtosis es significativo, respecto del de una distribución normal.

Tabla 4. Estadística descriptiva para los residuales de modelo ARMA de la demanda desestacionalizada y sin tendencia de energía mensual.

\begin{tabular}{lc}
\hline \multicolumn{1}{c}{ Estadístico } & $\begin{array}{c}\text { Demanda de energía } \\
(\mathbf{G W h})\end{array}$ \\
\hline \hline Mínimo & -10.6 \\
Mediana & -0.1 \\
Media & 0.0 \\
Máximo & 7.4 \\
Desv. Estándar & 2.8 \\
Sesgo & -0.2
\end{tabular}


Percentil $5 \quad-4.2$

Percentil $10 \quad-3.1$

Percentil 95 4.5

Percentil $90 \quad 3.2$

Percentil 99

Test sesgo normal: $\mathrm{T}=-0.16, \mathrm{p}$-value $=0.3245$

Test curtosis normal: $\mathrm{T}=4.1974, \mathrm{p}$-value $=0.0055$

Fuente: Elaboración propia.

En el Gráfico 4 se observa un mejor ajuste de la pdf Gram-Charlier que la pdf normal, en las colas de la distribución de frecuencia de los residuales estándar. El área gris, corresponde a la distribución de frecuencia suavizada, la línea continua a la distribución normal y la línea punteada a la función de densidad de probabilidad Gram-Charlier.

Gráfico 4. Ajuste de los residuales estándar del modelo a la distribución normal y la distribución GramCharlier.

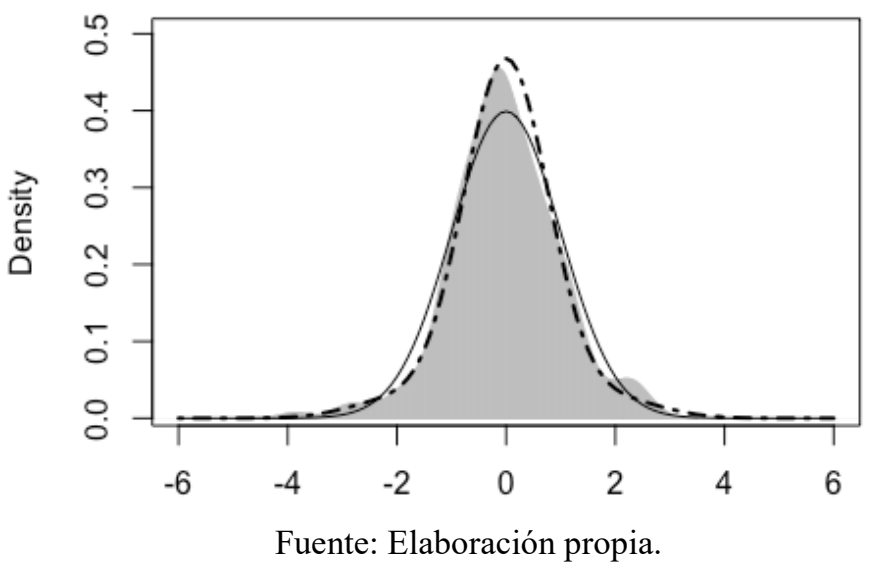

Por su parte, el Gráfico 5 muestra la función de probabilidad acumulada de los residuales del modelo ARMA. Se observa cómo al lado izquierdo de la mediana para cierto valor de la demanda la probabilidad acumulada es mayor para la distribución normal, pero en la cola izquierda, la pdf GCH y normal se cruzan y de esta manera, la probabilidad acumulada para cierto valor de la demanda en la cola izquierda, es mayor para la pdf GCH que para la pdf normal.

La función de probabilidad acumulada muestra como al lado izquierdo de la mediana para cierto valor de la demanda la probabilidad acumulada es mayor para la distribución normal, pero en la cola izquierda, la pdf GCH y normal se cruzan y de esta manera, la probabilidad acumulada para cierto valor de la demanda en la cola izquierda, es mayor para la pdf GCH que para la pdf normal. 
Gráfico 5. Probabilidad acumulada de las distribuciones normal y Gram-Charlier de los residuales del modelo ARMA.

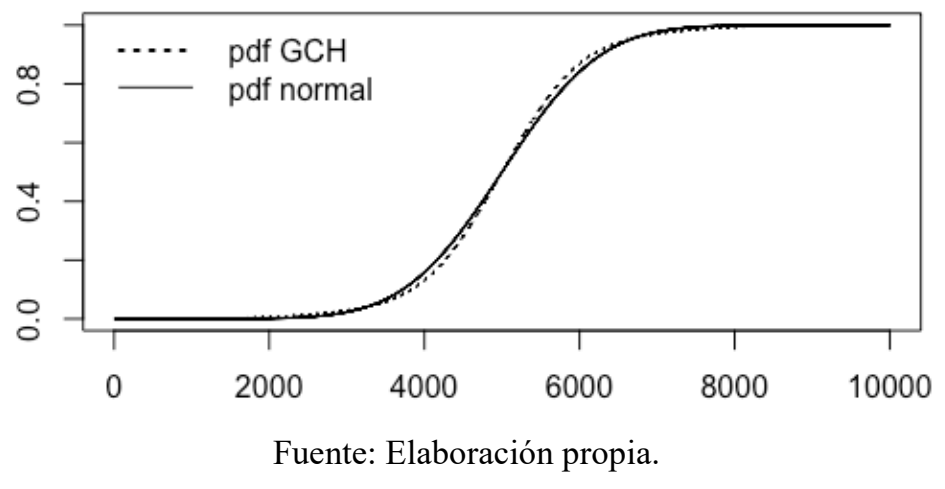

La Tabla 5 presenta los resultados del parámetro d4 ajustado a partir de la función de densidad SNP. Este parámetro captura el exceso de curtosis de la serie y estadísticamente resulta altamente significativo. Es de anotar que el parámetro de sesgo no resulta significativo.

Tabla 5. Parámetros ajustados a partir de la función de densidad SNP.

\begin{tabular}{|c|c|c|c|c|}
\hline & Estimado & Std & $\mathrm{t}$ & $\mathrm{p}$ value \\
\hline $\mathrm{d} 4$ & 0.0575002 & 0.0196507 & 2.92612 & 0.0034322 \\
\hline AIC SNP & 589.8019 & & & \\
\hline AIC Norm & 601.7897 & & & \\
\hline
\end{tabular}

Fuente: Elaboración propia.

\subsection{Aplicación de resultados y prueba de backtesting.}

En el Gráfico 6 se observa cómo el intervalo según la distribución Gram-Charlier es en general más amplio que el intervalo obtenido según la distribución normal, lo que indica que el riesgo de baja demanda es mayor si se mide con la distribución Gram-Charlier. La parte superior muestra el intervalo para toda la muestra. La parte inferior muestra el detalle entre enero de 2016 y abril de 2018.

Gráfico 6. Intervalo estimado con el $99 \%$ de probabilidad según la pdf GCH y la pdf normal.

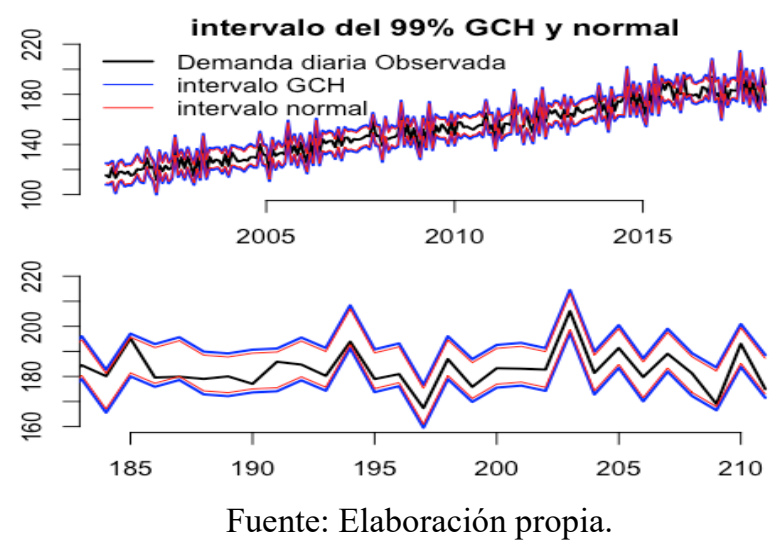


Una de las medidas de riesgo más empleadas en los mercados financieros es el valor en riesgo, o VaR por su sigla en inglés (Value at Risk), que mide la máxima pérdida esperada para un determinado horizonte de tiempo y un nivel de significancia, bajo condiciones normales de mercado. Según Hull (2002, p. 378) el VaR permite realizar afirmaciones como "estamos seguros en $(1-\alpha) \%$ de que no perderemos más de X dólares en los próximos $h$ días". Esta misma idea puede ser aplicada para la demanda de energía eléctrica tanto para el caso de la estimación de escenarios de máxima como de mínima demanda. A continuación, se presenta una aplicación de los resultados obtenidos y el backtesting o calibración que se aplica generalmente a los modelos VaR pero en este caso a la estimación del peor escenario de demanda desde el punto de vista del productor y comercializador. El concepto de VaR puede suscribirse en el concepto de Medida de Riesgo de Cuantil (Quantile Risk Metrics) que se define para algún número real $\alpha$ entre $0 \mathrm{y} 1$, como un cuantil x $\alpha$ de la distribución de una variable aleatoria continua $\mathrm{X}$ tal que:

$$
\mathrm{P}\left(\mathrm{X}<\mathrm{X}_{\alpha}\right)=\alpha
$$

Para medir la demanda diaria mínima esperada en un mes determinado se utiliza la estimación de un cuantil de la cola. En este caso se emplea un cuantil $\alpha$ bajo el que se acumula el $0.5 \%$ de la probabilidad. Dado que el intervalo de confianza es del 99.5\% (1- $\alpha$ a la derecha), se espera que la proporción $p$ de valores de la demanda diaria que son menores al cuantil $0.5 \%$ sea menor que $\alpha$.

Con el fin de evaluar la hipótesis nula de que $p=\alpha$, se puede emplear el estadístico $t_{U}$ de Kupiec (1995), cuya expresión corresponde a:

$$
t_{U}=\frac{\hat{p}-\alpha}{\sqrt{\frac{\hat{p}(1-\hat{p})}{H}}} .
$$

donde:

$\hat{p}=$ proporción de excepciones observadas

$H=$ número total de predicciones (o casos)

Kupiec (1995) demostró que ese estadístico sigue una distribución $t$ con $H-1$ grados de libertad. Así se rechazará la hipótesis nula, que establece que el modelo provee una cobertura (no condicional) deseada, si el valor absoluto de $t_{U}$ es mayor que el $t$ crítico asociado con una probabilidad $\alpha / 2$ y $H-1$ grados de libertad (en valor absoluto).

\begin{tabular}{ccc}
\hline & $\begin{array}{c}\text { Ajuste Distribución } \\
\text { Gram-Charlier }\end{array}$ & $\begin{array}{c}\text { Ajuste Distribución } \\
\text { Normal }\end{array}$ \\
\hline \hline Proporción de excepciones $(\widehat{\boldsymbol{p}})$ & 0.004739336 & 0.01895735 \\
Estadístico $\boldsymbol{t}_{\boldsymbol{U}}$ de Kupiec & -0.0551308 & 1.486659 \\
Valor $\boldsymbol{t}$ crítico & 0.9560867 & 0.1386047 \\
\hline
\end{tabular}

En este caso tenemos que el valor absoluto del estadístico $t_{U}$ de Kupiec es menor que el valor $t$ crítico para el ajuste a la distribución Gram-Charlier y mayor en el caso de la distribución normal. Entonces, se puede aceptar la hipótesis nula de que el modelo provee la cobertura deseada en el caso del ajuste a la distribución Gram-Charlier, pero se rechaza para el caso del ajuste a la distribución normal. 


\section{Conclusiones.}

En este trabajo se propone un modelo de demanda de energía eléctrica basado en métodos de series de tiempo y estadística semi-noparamétrica (SNP), que permite conocer no solo el valor esperado de la demanda sino también su distribución de probabilidad de manera que mediante el cálculo de métricas como la Medida de Riesgo de Cuantil (Quantile Risk Metrics) se puedan tomar decisiones basadas en valores extremos menos o más favorables que el valor esperado.

El modelo proporciona estimaciones de parámetros de la estructura temporal del valor esperado de largo plazo de la demanda diaria promedio, tal como lo es el parámetro estimado de tendencia, los efectos mensuales y otros efectos estacionales que se pueden capturar a través de términos de modelos autorregresivos y de medias móviles. Tomando como caso el mercado colombiano, en particular, se puede concluir que la demanda diaria promedio crece $0.35 \mathrm{GWh}$ por mes ( $4.2 \mathrm{GWh}$ por año). El mes de mayor demanda es agosto y el de menor demanda es febrero. Los modelos estacionales de variables dummy proporcionan parámetros que miden directamente la diferencia promedio en la demanda de cada mes.

Partiendo del modelo de valor esperado propuesto en este artículo, se puede concluir que la distribución de probabilidad de la demanda diaria promedio de energía eléctrica en Colombia es leptocúrtica. Es decir, los eventos extremos ocurren con mayor frecuencia que aquellos en los que se asume una distribución normal. De esta manera, el supuesto de distribución gaussiana conlleva a la subvaloración del riesgo en términos de la frecuencia de ocurrencia de valores extremos.

Los resultados muestran que la distribución de tipo SNP tiene un mejor desempeño para la modelización de la incertidumbre de la demanda de energía eléctrica respecto a la distribución normal (que se encuentra anidada en la SNP). Se observa que este enfoque permite capturar la leptocurtosis de la distribución de probabilidad de la demanda diaria.

Finalmente, los modelos basados en Medidas de Riesgo de Cuantil, permiten tomar decisiones teniendo en cuenta el apetito al riesgo, a través del parámetro $\alpha$ que es proporcional al riesgo que se corre en la toma de decisiones. Para trabajos futuros, se recomienda a los investigadores continuar aplicando las técnicas de estadística SNP a problemas relacionados con la demanda de energía eléctrica. Así como explorar su aplicación a diferentes variables que influyan en este mercado.

\section{Financiación}

Los autores agradecen la financiación brindada por el Instituto Tecnológico Metropolitano - ITM bajo el proyecto de investigación titulado "Valoración de parques eólicos para diversificar la canasta energética de Colombia". También se agradece a la Universidad EAFIT [Project 828-000019].

\section{Referencias}

Andalib, A., \& Atry, F. (2009). Multi-step ahead forecasts for electricity prices using NARX: a new approach, a critical analysis of one-step ahead forecasts. Energy Conversion Management, 50(3), 739-747. https://doi.org/10.1016/j.enconman.2008.09.040

Beenstock, M., Goldin, E., \& Nabot, D. (1999). The demand for electricity in Israel. Energy Economics, 21(2), 168-183. 
Bentzen, J., \& Engsted, T. (1993). Short- and long-run elasticities in energy demand: a cointegration approach. Energy Economics, 15(1), 9-16.

Bentzen, J., \& Engsted, T. (2001). A revival of the autoregressive distributed lag model in estimating energy demand relationships. Energy, 26(1), 45-55.

Bianco, V., Manca, O., \& Nardini, S. (2009). Electricity consumption forecasting in Italy using linear regression models. Energy, 34(9), 1413-1421. https://doi.org/10.1016/j.energy.2009.06.034

Bollerslev, T., \& Wooldridge, J. (1992). Quasi-maximum likelihood estimation and inference in dynamic models with time-varying covariances. Econometric Reviews, 11, 143-172.

Brunner, A.D. (1992). Conditional asymmetries in real GNP: A Seminonparametric Approach. Journal of Business \& Economic Statistics, 10(1), 65-72. https://doi.org/10.2307/1391805

Callaway, D.S. (2010). Sequential Reliability Forecasting for Wind Energy: Temperature Dependence and Probability Distributions. IEEE Transactions on Energy Conversion, 25(2), 577-585. https://doi.org/ 10.1109/TEC.2009.2039219.

Cortés, L.M., Mora-Valencia, A., \& Perote, J. (2018). Retrieving the implicit risk neutral density of WTI options with a semi-nonparametric approach. The North American Journal of Economics and Finance. In press. https://doi.org/10.1016/j.najef.2018.10.010

Debnath, K.B., \& Mourshed, M. (2018). Forecasting methods in energy planning models. Renewable and Sustainable Energy Reviews, 88(C), 297-325.

Del Brio, E., \& Perote, J. (2012). Gram-Charlier densities: Maximum likelihood versus the method of moments. Insurance: Mathematics and Economics, 51(3), 531-537. https://doi.org/ 10.1016/j.insmatheco.2012.07.005

Domeett, G. (2015). Análisis de los determinantes del cambio de la demanda de energía eléctrica en la ciudad de Neuquén. Ciencias Administrativas, 3(6), 1-15. https://revistas.unlp.edu.ar/CADM/article/view/1541

Ediger, V., \& Akar, S. (2007). ARIMA forecasting of primary energy demand by fuel in Turkey. Energy Policy, 35, 1701-1708.

El-Desouky, A.A. \& El-Kateb, M.M. (2000). Hybrid adaptive techniques for electric-load forecast using ANN and ARIMA. IEE Proceedings: Generation, Transmission and Distribution, 147(4), 213-217.

Engle, R.F., Granger, C.W.J., \& Hallman, J.J. (1989). Merging short-and long-run forecasts: an application of seasonal cointegration to monthly electricity sales forecasting. Journal of Econometrics, 40(1), 45-62.

Erdougdu, E. (2007). Electricity demand analysis using cointegration and ARIMA modelling: A case study of Turkey. Energy Policy, 35, 1129-1146.

Fouquet, R., Hawdon, D., Pearson, P., Robinson, C., \& Stevens, P. (1993). The SEEC United Kingdom energy demand forecast. Surrey Energy Economics Centre (SEEC): Occasional Paper 1, Department of Economics, University of Surrey, Guildford, UK. 
González-Romera, E., Jaramillo-Morán, M.A., \& Carmona-Fernández, D. (2006). Monthly electric energy demand forecasting based on trend extraction. IEEE Transactions Power Systems, 21(4), 1946-1953. https://doi.org/10.1109/TPWRS.2006.883666

Hull, J.C. (2002). Introducción a los Mercados de Futuros y Opciones. Boston: Prentice Hall.

Hunt, L.C., \& Witt, R. (1995). An analysis of UK energy demand using multivariate cointegration. Surrey Energy Economics Centre (SEEC): Discussion Paper No: 86, Department of Economics, University of Surrey, Guildford, UK.

Hussain, A., Rahman, M., \& Memon, J.A. (2016). Forecasting electricity consumption in Pakistan: the way forward. Energy Policy, 90, 73-80.

Ioannou, A., Angus, A., \& Brennan, F. (2017). Risk-based methods for sustainable energy system planning: A review. Renewable and Sustainable Energy Reviews, 74, 602-615.

Jondeau, E., \& Rockinger, M. (2001). Gram-Charlier densities. Journal of Economic Dynamics \& Control, 25(10), 1457-1483. https://doi.org/10.1016/s0165-1889(99)00082-2

Kupiec, P. (1995). Techniques for verifing the accuracy of risk measurement models. The Journal of Derivatives, 3, 71-84.

León, A., Mencia, J., \& Sentana, E. (2007). Parametric Properties of Semi-Nonparametric Distributions, with Applications to Option Valuation. Documentos de trabajo No 0707. Madrid, España: Banco de España.

Lucia, J.J., \& Schwartz, E.S. (2002). Electricity prices and power derivatives: Evidence from the Nordic power exchange. Review of Derivatives Research, 5(1), 5-50. https://doi.org/10.1023/A:1013846631785

Mauleon, I., \& Perote, J. (2000). Testing densities with financial data: An Empirical comparison of the Edgeworth-Sargan density to the Student's t. The European Journal of Finance, 6(2), 225-239. https://doi.org/10.1080/13518470050020851

Melikoglu, M. (2013). Forecasting Turkey's natural gas demand between 2013 and 2030. Renew Sustain Energy Reviews, 22, 393-400.

Nasr, G., Badr, E., \& Dibeh, G. (2000). Econometric modelling of electricity consumption in postwar Lebanon. Energy Economics, 22(6), 627-640.

Ñiguez, T.-M., \& Perote, J. (2011). Forecasting havy-tailed densities with positive Edgeworth and Gram-Charlier expansions. Oxford Bulletin of Economics and Statics, 74(4), 600-627. https://doi.org/10.1111/j.1468-0084.2011.00663.x

UPME (2016). Seguridad energética para Colombia. Entregable 3: Informe Final. https://bdigital.upme.gov.co/bitstream/001/1314/1/Seguridad\%20Energ\%C3\%A9tica\%20UPMECIDET\%20Entrega\%20Final.pdf

Trespalacios, A., Cortés, L., \& Perote, J. (2020). Uncertainty in electricity markets from a seminonparametric approach. Energy Policy, 137, 111091.

Tsay, R.S. (2005). Analysis of Financial Time Series. Vol. 543. Chicago: John Wiley \& sons. 
Yuan, C., Liu, S., \& Fang, Z. (2016). Comparison of China's primary energy consumption forecasting by using ARIMA (the autoregressive integrated moving average) model and $\operatorname{GM}(1,1)$ model. Energy, 100, 384-390. 